\title{
Evaluating vaspin and adiponectin in postmenopausal women with endometrial cancer
}

\author{
Serpil Erdogan, Sevilay Sezer, Eralp Baser ${ }^{1}$, Ozlem Gun-Eryilmaz', Tayfun Gungor', \\ Sema Uysal and Fatma Meric Yilmaz \\ Department of Clinical Biochemistry, Numune Training and Research Hospital, Ankara, Turkey \\ 'Department of Gynecologic Oncology, Dr Zekai Tahir Burak Women' Health, Education, and Research Hospital, \\ Ankara, Turkey
}

Correspondence should be addressed to $S$ Erdogan

Email

drsturhan@yahoo.com

\begin{abstract}
Insulin resistance is a well-documented risk factor for the development of endometrial cancer. Adiponectin and vaspin are insulin-sensitizing proteins that are secreted from adipose tissue. A clear association between serum levels of adipokines and endometrial cancer has yet to be established. The study group consisted of postmenopausal women with confirmed endometrial cancer, whereas patients with benign endometrial conditions constituted the control group. The two groups were compared in terms of insulin resistance and serum levels of adiponectin and vaspin. A total of 60 patients with confirmed endometrial cancer and 70 controls with benign endometrial conditions (polyps and atrophy) were enrolled. Median homeostasis model assessment of insulin resistance value was significantly higher in the study group compared with the control group ( 2.93 vs 1.27 , $P<0.0001$ ), whereas mean quantitative insulin sensitivity check index value was significantly lower $(0.33 \pm 0.02$ vs $0.37 \pm 0.37, P<0.0001)$. Median values for both adiponectin and vaspin were significantly lower in patients with endometrial cancer compared with the control group (4.09 vs $17.13 \mu \mathrm{g} / \mathrm{ml}, P<0.0001$ and 0.21 vs $0.39 \mathrm{ng} / \mathrm{ml}, P<0.0001$ respectively). Low levels of both adiponectin and vaspin were found to be significantly associated with an increased risk for endometrial cancer. Following adjustment for confounding factors, the respective odds ratios for endometrial cancer in patients in the first tertile compared with those in the third tertile were $10.80(2.76-42.24 ; P=0.001)$ and $13.23(2.94-59.64 ; P=0.001)$. Our results show that lower levels of circulating adiponectin and vaspin levels are associated with an increased risk of developing endometrial cancer.
\end{abstract}
Key Words
- adiponectin
- vaspin
- insulin resistance
- endometrial cancer

Endocrine-Related Cancer (2013) 20, 669-675

\section{Introduction}

Cancer is the second leading cause of death in developed countries, behind heart disease (Jemal et al. 2005). It is estimated that in the USA alone, nearly 580350 individuals will have died from cancer by 2013, corresponding to almost 1600 deaths per day. Corporal uterine cancer, which is the most commonly diagnosed gynecological cancer, is responsible for the death of one fifth of women with endometrial cancer (Siegel et al. 2013).

The prevailing theory regarding the factors thought to be responsible for the development of endometrial cancer is known as the 'unopposed estrogen' hypothesis, where the mitogenic effects of estrogens are insufficiently 
counterbalanced by progesterone (Kaaks et al. 2002). However, the correlation noted between obesity and endometrial cancer cannot be accounted for by the risk posed by elevated levels of endogenous estrogens alone. It has been postulated that BMI and other obesity-related factors such as insulin resistance may contribute to an increased risk for endometrial cancer (Soliman et al. 2006).

Adiponectin, a 244-amino acid protein, is one of the earliest adipokines secreted from adipose tissue to be discovered (Maeda et al. 1996, Arita et al. 1999). It sensitizes peripheral tissues to insulin, and decreased levels result in insulin resistance, which is associated with an increased risk of obesity and type 2 diabetes mellitus (Petridou et al. 2003). Several studies have demonstrated the presence of a link between adiponectin and endometrial cancer (Petridou et al. 2003, Dal Maso et al. 2004, Soliman et al. 2006, Cust et al. 2007, Ashizawa et al. 2010.).

Visceral adipose tissue-derived serine protease inhibitor (Vaspin) is a $50 \mathrm{kDa}$ adipokine, which was first discovered in visceral white adipose tissue of Otsuka Long-Evans Tokushima rats used as an animal model of obesity and type 2 diabetes (Hida et al. 2005). Recent studies have suggested that changes in vaspin levels may be linked with diabetes mellitus, metabolic syndrome, obesity, and impaired insulin sensitivity (Hida et al. 2005, Li et al. 2011), all of which have been described as risk factors for endometrial cancer (Schmandt et al. 2011). Although the association between adiponectin and endometrial cancer has been evaluated in several studies, data on the role of vaspin have yet to be published. The aim of this study was to determine whether changes in adiponectin and vaspin levels were associated with an increased risk of endometrial cancer.

\section{Subjects and methods}

This prospective cross-sectional controlled study was conducted in the Department of Gynecologic Oncology at Zekai Tahir Burak Women's Health Education and Research Hospital in Ankara, Turkey, with the approval of the local ethics committee. Postmenopausal women with histologically or cytologically confirmed endometrial cancer who were evaluated between April 2012 and January 2013 were approached for inclusion in the study, and consenting patients were screened for eligibility. The control group consisted of consenting patients who presented during the same period with benign endometrial conditions such as endometrial polyps and atrophic endometrium.

Patients with a previous history of cancer, polycystic ovary syndrome, atherosclerotic disease, hypertension, asthma, diabetes mellitus, heart failure, peripheral vascular disease, hematological disease, and autoimmune disorders were excluded from the study. Other exclusion criteria included alcohol consumption, active smoking, current use of any medication such as oral contraceptives or hormone replacement treatment, impaired liver or renal function test, and a history of a hysterectomy.

Information on demographics, level of education, and family history of endometrial cancer were recorded for all participants, followed by a careful physical examination including anthropometric measurements. BMI was calculated by dividing body weight in kilograms by the square of a patient's height in meters.

All blood samples were obtained by venipuncture of the antecubital vein between 0800 and $0900 \mathrm{~h}$ following an overnight fast of $8-12 \mathrm{~h}$. The obtained sample for each patient was transferred to an EDTA tube and a standard biochemistry tube that was centrifuged immediately. Levels of $\mathrm{HbA1c}$ were determined using a turbidimetric inhibition immunoassay (Roche/Hitachi Modular P analyzer) for whole blood. Serum glucose was assayed using the glucose oxidase method by an autoanalyzer (Roche/Hitachi Modular P analyzer). Insulin and CA 125 levels were measured by a chemiluminescent microparticle immunoassay on an ADVIA Centaur analyzer (Siemens AG, Erlangen, Germany). The remaining sera were stored at $-80^{\circ} \mathrm{C}$ for the evaluation of serum levels of adiponectin and vaspin at the end of the study. Serum adiponectin (eBioscience; Bender MedSystems, Vienna, Austria; Lot No. 77738005) and vaspin (BioVendor-Laboratorní medicína a.s; Brno, Czech Republic; Lot No. E12-077S4) levels were measured by ELISA with an Epoch system (BioTek Instruments, Winooski, VT, USA). The minimal detectable concentrations of adiponectin and vaspin were estimated to be $1.0 \mu \mathrm{g} / \mathrm{ml}$ and $0.01 \mathrm{ng} / \mathrm{ml}$ respectively. The intra-assay coefficients of variation for serum adiponectin and vaspin were 4.2 and $7.6 \%$ respectively, and the inter-assay coefficients of variation for serum adiponectin and vaspin were 3.1 and $7.7 \%$ respectively.

The homeostasis model assessment of insulin resistance (HOMA-IR) and quantitative insulin sensitivity check index (QUICKI) were calculated from fasting insulin (FINS) and fasting blood glucose (FBG) levels using the following equations (Matthews et al. 1985, Katz et al. 2000):

$$
\begin{aligned}
& \text { HOMA-IR }=\frac{\text { FINS }(\mathrm{mU} / \mathrm{l}) \times \text { FBG }(\mathrm{mmol} / \mathrm{l})}{22.5} \\
& \text { QUICKI }=\frac{1}{\log (\mathrm{FINS}(\mathrm{mU} / \mathrm{l}))+\log (\mathrm{FBG}(\mathrm{mg} / \mathrm{dl}))}
\end{aligned}
$$

Published by Bioscientifica Ltd 


\section{Statistical analysis}

Statistical analyses were performed using the PASW Statistics 18 software (IBM Corporation, Armonk, New York, USA). Normality of distribution was evaluated using the Kolmogorov-Smirnov test. Comparisons of variables with a normal distribution were made using the Student's $t$-test, and values were provided as mean \pm s.D. For parameters with an abnormal distribution, the MannWhitney $U$ test was used for comparisons, and values were given as median (minimum-maximum).

Conditional logistic regression, which preserved the matching of cases and controls, was used to estimate the odds ratios (ORs) and the $95 \%$ CIs for the associations between the serum vaspin levels and the endometrial cancer risk. To develop the logistic regression models, the serum levels of vaspin and adiponectin were categorized into tertiles based on the distribution of values in the control group. A $P$ value of $<0.05$ was considered indicative of statistical significance.

\section{Results}

A total of 60 patients with histologically or cytologically confirmed endometrial cancer, along with 70 controls, were included in this study, the demographic characteristics of which have been summarized in Table 1. Both mean age and BMI were significantly higher in the study group compared with the controls ( 56.57 vs 49.70 years; $31.12 \mathrm{vs} 27.49 \mathrm{~kg} / \mathrm{m}^{2}$ ). Level of education was also lower in patients with endometrial cancer compared with the controls (5 vs 8 years). None of the participants gave a family history of endometrial cancer.

In the study group, 49 patients (81.67\%) had endometrioid adenocarcinoma, and according to the criteria of the International Federation of Gynecology and Obstetrics (FIGO), 37 (61.7\%) had stage IA disease, 14 (23.3\%) had stage IB, two (3.3\%) had stage II, one (1.7\%) had stage IIIA, two (3.3\%) had stage IIIC1, three (5.0\%) had stage IIIC2, and one $(1.7 \%)$ patient had stage IVA disease.

All participants had an HbA1c value of $<6.5 \%$ (4.62$6.41 \%)$. Mean HOMA-IR score was significantly higher in patients with endometrial cancer compared with the control group (2.93 vs $1.27 ; P<0.0001)$, whereas QUICKI values were significantly lower in the study group than in the controls ( 0.33 vs $0.37 ; P<0.0001)$. The endometrial cancer group also had a significantly higher mean CA 125 level compared with the control group (12.95 vs $6.30 \mathrm{IU} / \mathrm{ml} ; P<0.0001)$.

Median values for both adiponectin and vaspin were significantly lower in patients with endometrial cancer compared with the control group (4.09 vs $17.13 \mu \mathrm{g} / \mathrm{ml}$, $P<0.0001$ and 0.21 vs $0.39 \mathrm{ng} / \mathrm{ml}, P<0.0001$ respectively). Results of biochemical assays along with HOMA-IR and QUICKI scores have been summarized in Table 2.

In a subset analysis, we categorized the serum vaspin and adiponectin levels into three groups based on the tertile values in the control group. Lower adiponectin levels were found to be significantly associated with an increased risk of endometrial cancer. Compared with subjects in the highest tertile for adiponectin, the OR of endometrial cancer for the subjects in the first tertile was 10.64 (3.61-31.40). Lower vaspin levels were found to be significantly associated with an increased risk of endometrial cancer. Compared with subjects in the highest tertile of vaspin, the OR of endometrial cancer for the subjects in the first tertile was 17.04 (4.65-62.44). Furthermore, the association of the adiponectin and vaspin levels for the first tertile with the risk of endometrial cancer remained significant after adjusting for confounding factors such as age, BMI, HOMA-IR, and QUICKI. Table 3 shows the risk of endometrial cancer in relation to the serum vaspin and adiponectin levels.

\section{Discussion}

Endometrial cancer is one of the most important causes of death in developed countries (Jemal et al. 2005). It has been suggested that obesity may be linked with an increased risk of developing endometrial cancer. Four hormonal mechanisms have been postulated; increased estrogen levels via conversion of circulating androgens in

Table 1 Demographic characteristics for cases and controls

\begin{tabular}{lcc} 
& & Cases $(n=60)$ \\
\hline Age (years) (mean \pm s.D.) & & $56.57 \pm 9.05$ \\
BMI (kg/m²) (mean \pm s.D.) & & $31.12 \pm 4.18$ \\
$\begin{array}{c}\text { Years in school (median } \\
(\text { min-max)) }\end{array}$ & $5(5-8)$ \\
\hline
\end{tabular}

min, minimum value; max, maximum value.

\begin{tabular}{|c|c|}
\hline Controls $(n=70)$ & $P$ value \\
\hline $\begin{array}{c}49.70 \pm 7.59 \\
27.49 \pm 3.22 \\
8(5-11)\end{array}$ & $\begin{array}{l}<0.0001 \\
<0.0001 \\
<0.0001\end{array}$ \\
\hline
\end{tabular}

http://erc.endocrinology-journals.org DOI: 10.1530/ERC-13-0280
(C) 2013 Society for Endocrinology Printed in Great Britain
Published by Bioscientifica Ltd. 
Table 2 Serum adipokines, CA 125 levels, and markers of insulin resistance in the cases and controls

\begin{tabular}{|c|c|c|c|}
\hline & Cases $(n=60)$ & Controls $(n=70)$ & $P$ value \\
\hline HOMA-IR (median (min-max)) & $2.93(0.87-8.20)$ & $1.27(0.23-22.28)$ & $<0.0001$ \\
\hline QUICKI (mean \pm s.D.) & $0.33 \pm 0.02$ & $0.37 \pm 0.37$ & $<0.0001$ \\
\hline CA 125 (IU/ml) (median (min-max)) & $12.95(2.5-354.1)$ & $6.30(0.60-25.00)$ & $<0.0001$ \\
\hline Adiponectin $(\mu \mathrm{g} / \mathrm{ml})($ median $(\min -\mathrm{max}))$ & $4.09(0.67-43.55)$ & $17.13(2.59-108.69)$ & $<0.0001$ \\
\hline Vaspin (ng/ml) (median (min-max)) & $0.21(0.11-0.54)$ & $0.39(0.34-1.30)$ & $<0.0001$ \\
\hline
\end{tabular}

HOMA-IR, the homeostasis model assessment of insulin resistance; QUICKI, quantitative insulin sensitivity check index; min, minimum value; max, maximum value.

peripheral adipocytes, increased adrenal and ovarian secretion of androgens, increased free steroid hormone levels secondary to decreased sex hormone binding globulin production, and decreased progesterone production in premenopausal women secondary to decreased ovulation (Modesitt et al. 2012). It remains a fact that not all obese women develop endometrial cancer, and not all patients with endometrial cancer are obese (Modesitt et al. 2012). The role of other confounding factors in the carcinogenesis of endometrial cancer is currently under investigation.

Insulin resistance, which may be defined as a decreased sensitivity or responsiveness to the metabolic actions of insulin, was first identified nearly 80 years ago (Muniyappa et al. 2008). Although the hyperinsulinemic-euglycemic glucose clamp technique, which was originally described by DeFronzo et al. (1979), is considered by the American Diabetes Association to be the 'gold standard' for quantifying IR (American Diabetes Association 1998) its complexity and associated high cost have led to the development of simpler alternative methods such as HOMA-IR and QUICKI. HOMA-IR is considered a reliable indicator of insulin resistance whereas QUICKI provides critical information regarding insulin sensitivity (Matthews et al. 1985, Katz et al. 2000, Wallace et al. 2004). Although both scores have been used extensively in epidemiological studies and clinical research, QUICKI remains the best indicator among the simple surrogate indices for insulin sensitivity/resistance (Muniyappa et al. 2008).

Insulin resistance is a state of perpetually elevated plasma insulin levels, even in the fasting state (Kaaks et al. 2002). Chronic hyperinsulinemia has been established as an important risk factor for endometrial cancer (Schmandt et al. 2011), which has been attributed to a mitogenic effect via endometrial insulin receptors' direct enhancement of tumor formation (Nagamani \& Stuart 1998, Kaaks et al. 2002). Estrogen has also been implicated as a major culprit in endometrial carcinogenesis, due to its proliferative effects on endometrial tissue facilitated by an increase in local levels of insulin-like growth factor 1 (IGF1). Progesterone, on the other hand, is widely accepted as a protective factor against endometrial carcinogenesis due to its antiproliferative effects on endometrial tissue by promoting a local increase in IGF binding protein 1 (IGFBP) synthesis. Insulin manifests its carcinogenic characteristics by increasing the bioactivity of IGF1 while also downregulating the synthesis of IGFBP1 in endometrial tissue (Murphy \& Ghahary 1990, Rutanen et al. 1994). Furthermore, chronic hyperinsulinemia may lead to ovarian hyperandrogenism, and increased

Table 3 Logistic regression analysis of risk factors and endometrial cancer

\begin{tabular}{|c|c|c|c|c|c|c|}
\hline Tertiles & $\begin{array}{l}\text { Cases } \\
(n=60)\end{array}$ & $\begin{array}{c}\text { Controls } \\
(n=70)\end{array}$ & OR $(95 \% \mathrm{Cl})$ & $P$ value & $\begin{array}{l}\text { Adjusted } \mathbf{O R}^{\mathbf{a}} \\
\quad(95 \% \mathrm{Cl})\end{array}$ & $P$ value \\
\hline \multicolumn{7}{|c|}{ Adiponectin $(\mu \mathrm{g} / \mathrm{ml})$} \\
\hline$<10.91$ & 51 & 23 & $10.64(3.61-31.40)$ & $<0.0001$ & $10.80(2.76-42.24)$ & 0.001 \\
\hline $10.91-21.14$ & 4 & 23 & $0.84(0.20-3.50)$ & 0.805 & $0.62(0.12-3.37)$ & 0.582 \\
\hline$>21.14$ & 5 & 24 & $1^{\mathrm{b}}$ & & $1^{\mathrm{b}}$ & \\
\hline \multicolumn{7}{|l|}{ Vaspin (ng/ml) } \\
\hline$<0.36$ & 49 & 23 & $17.04(4.65-62.44)$ & $<0.0001$ & $13.23(2.94-59.64)$ & 0.001 \\
\hline $0.36-0.42$ & 8 & 23 & $2.78(0.66-11.80)$ & 0.165 & $2.07(0.39-11.03)$ & 0.392 \\
\hline$>0.42$ & 3 & 24 & $1^{\mathrm{b}}$ & & $1^{\mathrm{b}}$ & \\
\hline
\end{tabular}

Serum vaspin and adiponectin were categorized by the tertile value in the control group. OR, odds ratio. Bold type indicates a statistically significant difference. ${ }^{a}$ ORs are estimated from multiple logistic regression analysis, adjusted by age, BMI, HOMA-IR and QUICKI.

${ }^{\mathrm{b}}$ Reference category.

http://erc.endocrinology-journals.org DOI: 10.1530/ERC-13-0280
(C) 2013 Society for Endocrinology Printed in Great Britain
Published by Bioscientifica Ltd. 
peripheral aromatization of androgens to estrogen may increase the risk of endometrial cancer, particularly in postmenopausal women (Kaaks et al. 2002).

In a case-control study from Japan, investigators managed to demonstrate a significant correlation between adiponectin and HOMA-IR, but not with FINS (Ashizawa et al. 2010). Recently, Modesitt et al. (2012) did not observe any difference between patients with and without cancer in terms of insulin levels. We did not encounter any study in the English literature in which HOMA-IR and QUICKI were evaluated in patients with endometrial cancer. Our study is the first to establish the presence of a significant association of higher HOMA-IR and lower QUICKI values, as predictors of insulin resistance and sensitivity, with an increased risk of endometrial cancer. Our results suggest a possible role for insulin resistance in endometrial carcinogenesis.

Adipose tissue, which has recently been classified as an endocrine organ, releases adipokines that have both antiinflammatory and proinflammatory characteristics (Schmandt et al. 2011). The first adipokine to be identified as a product of white adipose tissue was adiponectin, a 244-amino acid protein with a high structural homology to collagen VIII (Maeda et al. 1996, Arita et al. 1999, Takahashi et al. 2000). Ever since its discovery, adiponectin has been the focus of extensive research that has demonstrated significant effects on the inhibitory actions of insulin on hepatic glucose production via the activation of AMP-activated protein kinase (AMPK), an energy sensor that controls cellular metabolism (Wang et al. 2002, Long $\&$ Zierath 2006). Activation of AMPK in skeletal muscle, liver, and adipose tissue results in enhanced insulin sensitivity (Long \& Zierath 2006). In a study from Japan, administration of adiponectin to lipodystrophic mice was found to reduce insulin resistance (Yamauchi et al. 2001). Decreased adiponectin receptor 1 (AdipoR1)/AdipoR2 expression has also been shown to be closely associated with neoplastic growth of the endometrium. Investigators reported lower levels of AdipoR1 in endometrial adenocarcinoma patients with myometrial invasion or lymph node metastasis (Yamauchi et al. 2012). It has been postulated that adiponectin may prevent tumorigenesis by activating AMPK and inhibiting phosphatidylinositol 3-kinase (PI3K)/AKT/mTOR signaling via AdipoR1/2 (Schmandt et al. 2011). Subsequent studies have indeed managed to demonstrate the presence of an antitumorigenic effect of adiponectin in endometrial cancer patients. In a recent study on 146 postmenopausal female subjects with endometrial cancer and 150 control subjects, the reported mean serum adiponectin level was significantly lower in the cancer group $(P<0.0001)$, while a negative correlation between adiponectin and BMI was also observed. Compared with subjects in the lowest tertile, the ORs of endometrial cancer for the subjects in the third tertile was 0.5 (95\% CI 0.3-0.9) (Ashizawa et al. 2010). In another study from Greece, an increase in adiponectin levels by 1 s.D. was found to be associated with more than a 50\% reduction in the risk of endometrial cancer, even after adjustment for other potential confounders such as BMI (Petridou et al. 2003). In a study by Soliman et al. (2006), patients with endometrial cancer were found to have higher adiponectin levels than controls, and following adjustment to confounding factors, the OR of developing endometrial cancer in subjects in the lowest tertile was 10.5. Subgroup analyses in the same study revealed normal-weight women $\left(\mathrm{BMI} \leq 25 \mathrm{~kg} / \mathrm{m}^{2}\right)$ with serum adiponectin levels in the lowest tertile to have approximately a 20 -fold increased risk of developing endometrial cancer. Furthermore, investigators established that adiponectin level was inversely correlated with BMI in the control group but not in the endometrial cancer group. Two other European studies have also identified a low serum adiponectin level as an independent risk factor for carcinogenesis, after adjusting for other confounding factors such as age and BMI (Dal Maso et al. 2004, Cust et al. 2007). On the other hand, a conflicting result was reported in a recent pilot study, where authors did not observe a significant difference between patients with and without endometrial cancer in terms of serum adiponectin levels (Modesitt et al. 2012). Regardless, comparison of patients with endometrial cancer and those with benign endometrial conditions in our study revealed cancer patients to have significantly lower serum adiponectin levels (4.09 vs 17.13; $P<0.0001$ ), higher HOMA-IR values (2.93 vs 1.27 ; $P<0.0001)$, as an indicator of insulin resistance, and lower QUICKI values ( 0.33 vs $0.37 ; P<0.0001)$, as an estimate of insulin sensitivity. We established that low adiponectin levels were associated with a tenfold increased risk for endometrial cancer.

Vaspin, which is another adipokine made of 395 amino acids, shares $\sim 40 \%$ homology with alpha1 antitrypsin (Hida et al. 2005). In a recent study by Kadoglou et al. (2011), lower serum vaspin levels were observed in patients with angiographically confirmed coronary artery disease compared with age- and sexmatched healthy controls. Investigators also reported on an inverse relationship between vaspin and the number of significantly narrowed coronary arteries. Several studies have established that decreased serum levels and reduced

Published by Bioscientifica Ltd 
mRNA expression of vaspin in peripheral blood mononuclear cells are associated with an increased risk of developing carotid stenosis, coronary artery disease, and unstable angina pectoris, as well as other microvascular complications in patients with diabetes mellitus (Aust et al. 2009, Gulcelik et al. 2009, Li et al. 2011, Kobat et al. 2012).

Diminished expression of vaspin, which is an insulinsensitizing and anti-inflammatory molecule, has been shown to be associated with worsening of diabetes-related complications, with serum vaspin levels normalizing following treatment with insulin or pioglitazone (Hida et al. 2005, Phalitakul et al. 2011). Under hyperglycemia, vaspin leads to attenuated high glucose (HG)-induced phosphorylation of both insulin receptor and insulin receptor substrate (IRS)-1, while at the same time suppressing phosphorylation of IRS-2. This has been proposed as a novel mechanism by which vaspin offers protection against vascular injury brought on by HG-induced insulin resistance. Vaspin has also been shown to reduce HG-stimulated vascular smooth muscle cell proliferation and chemokinesis, in vitro, by preventing the activation of reactive oxygen species, MAPK and PI3K/Akt while also blocking insulin receptor signaling and the NF- $\mathrm{KB}$ signaling pathway (Li et al. 2013). It was concluded that the delaying effect of vaspin on vascular intimal hyperplasia could also play a possible role in the delay or prevention of carcinogenesis (Li et al. 2013). However, to date, no papers have been published regarding this matter, and there is a need for further studies investigating the pathogenic mechanisms involved in carcinogenesis, at a molecular level.

With this study, we managed to demonstrate a link between low serum vaspin levels and an increased risk of endometrial cancer, whereas low levels of adiponectin were found to be associated with a 13-fold increase in the risk of developing endometrial cancer. To the best of our knowledge, this is the first study to investigate the relationship between endometrium cancer and serum vaspin levels.

Although participants in this study were meticulously selected through several exclusion criteria, it is not without limitations. The number of consenting participants included was limited, and consequently, sufficient matching for BMI and age could not be performed. Furthermore, most of the patients $(\sim 85 \%)$ had early stage (stage IA and IB) disease. Additionally, HbA1c measurements were made for patients with endometrial cancer but not for participants with benign endometrial conditions.
In summary, we observed that endometrial cancer risk was significantly increased in women with lower serum vaspin and adiponectin levels, and this significance was independent of well-known risk factors such as age, BMI, HOMA-IR, and QUICKI. Although we investigated the role of these adipokines from an insulin resistance perspective, our results suggested that decreased vaspin and adiponectin levels may be associated with a currently undiscovered alternative mechanism in endometrial carcinogenesis, which should be clarified in future more comprehensive studies.

\section{Declaration of interest}

The authors declare that there is no conflict of interest that could be perceived as prejudicing the impartiality of the research reported.

\section{Funding}

This study was financially supported by grants from Ankara Numune Training and Research Hospital (Project number: 382/2012).

\section{References}

American Diabetes Association 1998 Consensus Development Conference on Insulin Resistance. 5-6 November 1997. Diabetes Care 21 310-314.

Arita Y, Kihara S, Ouchi N, Takahashi M, Maeda K, Miyagawa J, Hotta K, Shimomura I, Nakamura T, Miyaoka K et al. 1999 Paradoxical decrease of an adipose-specific protein, adiponectin, in obesity. Biochemical and Biophysical Research Communications 257 79-83. (doi:10.1006/bbrc. 1999.0255)

Ashizawa N, Yahata T, Quan J, Adachi S, Yoshihara K \& Tanaka K 2010 Serum leptin-adiponectin ratio and endometrial cancer risk in postmenopausal female subjects. Gynecologic Oncology 119 65-69. (doi:10.1016/j.ygyno.2010.07.007)

Aust G, Richter O, Rohm S, Kerner C, Hauss J, Kloting N, Ruschke K, Kovacs P, Youn BS \& Bluher M 2009 Vaspin serum concentrations in patients with carotid stenosis. Atherosclerosis 204 262-266. (doi:10.1016/j.atherosclerosis.2008.08.028)

Cust AE, Kaaks R, Friedenreich C, Bonnet F, Laville M, Lukanova A, Rinaldi S, Dossus L, Slimani N, Lundin E et al. 2007 Plasma adiponectin levels and endometrial cancer risk in pre- and postmenopausal women. Journal of Clinical Endocrinology and Metabolism 92 255-263. (doi:10.1210/jc.2006-1371)

Dal Maso L, Augustin LS, Karalis A, Talamini R, Franceschi S, Trichopoulos D, Mantzoros CS \& La Vecchia C 2004 Circulating adiponectin and endometrial cancer risk. Journal of Clinical Endocrinology and Metabolism 89 1160-1163. (doi:10.1210/jc.2003031716)

DeFronzo RA, Tobin JD \& Andres R 1979 Glucose clamp technique: a method for quantifying insulin secretion and resistance. American Journal of Physiology 237 E214-E223.

Gulcelik NE, Karakaya J, Gedik A, Usman A \& Gurlek A 2009 Serum vaspin levels in type 2 diabetic women in relation to microvascular complications. European Journal of Endocrinology 160 65-70. (doi:10.1530/EJE-08-0723)

Hida K, Wada J, Eguchi J, Zhang H, Baba M, Seida A, Hashimoto I, Okada T, Yasuhara A, Nakatsuka A et al. 2005 Visceral adipose tissue-derived 
serine protease inhibitor: a unique insulin-sensitizing adipocytokine in obesity. PNAS 102 10610-10615. (doi:10.1073/pnas.0504703102)

Jemal A, Ward E, Hao Y \& Thun M 2005 Trends in the leading causes of death in the United States, 1970-2002. Journal of the American Medical Association 294 1255-1259. (doi:10.1001/jama.294.10.1255)

Kaaks R, Lukanova A \& Kurzer MS 2002 Obesity, endogenous hormones, and endometrial cancer risk: a synthetic review. Cancer Epidemiology, Biomarkers \& Prevention 11 1531-1543.

Kadoglou NP, Gkontopoulos A, Kapelouzou A, Fotiadis G, Theofilogiannakos EK, Kottas G \& Lampropoulos S 2011 Serum levels of vaspin and visfatin in patients with coronary artery disease - Kozani study. Clinica Chimica Acta 412 48-52. (doi:10.1016/i.cca.2010.09.012)

Katz A, Nambi SS, Mather K, Baron AD, Follmann DA, Sullivan G \& Quon MJ 2000 Quantitative insulin sensitivity check index: a simple, accurate method for assessing insulin sensitivity in humans. Journal of Clinical Endocrinology and Metabolism 85 2402-2410. (doi:10.1210/jc. 85.7.2402)

Kobat MA, Celik A, Balin M, Altas Y, Baydas A, Bulut M, Aydin S, Dagli N, Yavuzkir MF \& Ilhan S 2012 The investigation of serum vaspin level in atherosclerotic coronary artery disease. Journal of Clinical Medicine Research 4 110-113. (doi:10.4021/jocmr841w)

Li HL, Peng WH, Cui ST, Lei H, Wei YD, Li WM \& Xu YW 2011 Vaspin plasma concentrations and mRNA expressions in patients with stable and unstable angina pectoris. Clinical Chemistry and Laboratory Medicine 49 1547-1554. (doi:10.1515/CCLM.2011.236)

Li H, Peng W, Zhuang J, Lu Y, Jian W, Wei Y, Li W \& Xu Y 2013 Vaspin attenuates high glucose-induced vascular smooth muscle cells proliferation and chemokinesis by inhibiting the MAPK, PI3K/Akt, and NF-кB signaling pathways. Atherosclerosis 228 61-68. (doi:10.1016/j. atherosclerosis.2013.02.013)

Long YC \& Zierath JR 2006 AMP-activated protein kinase signaling in metabolic regulation. Journal of Clinical Investigation 116 1776-1783. (doi:10.1172/JCI29044)

Maeda K, Okubo K, Shimomura I, Funahashi T, Matsuzawa Y \& Matsubara K 1996 cDNA cloning and expression of a novel adipose specific collagen-like factor, apM1 (AdiPose Most abundant Gene transcript 1). Biochemical and Biophysical Research Communications 221 286-289. (doi:10.1006/bbrc.1996.0587)

Matthews DR, Hosker JP, Rudenski AS, Naylor BA, Treacher DF \& Turner RC 1985 Homeostasis model assessment: insulin resistance and $\beta$-cell function from fasting plasma glucose and insulin concentrations in man. Diabetologia 28 412-419. (doi:10.1007/BF00280883)

Modesitt SC, Geffel DL, Via J \& Weltman A 2012 Morbidly obese women with and without endometrial cancer: are there differences in measured physical fitness, body composition, or hormones? Gynecologic Oncology 124 431-436. (doi:10.1016/j.ygyno.2011.11.013)

Muniyappa R, Lee S, Chen H \& Quon MJ 2008 Current approaches for assessing insulin sensitivity and resistance in vivo: advantages, limitations, and appropriate usage. American Journal of Physiology. Endocrinology and Metabolism 294 E15-E26. (doi:10.1152/ajpendo. 00645.2007)

Murphy LJ \& Ghahary A 1990 Uterine insulin-like growth factor-1: regulation of expression and its role in estrogen-induced uterine proliferation. Endocrine Reviews 11 443-453. (doi:10.1210/ edrv-11-3-443)

Nagamani M \& Stuart CA 1998 Specific binding and growth-promoting activity of insulin in endometrial cancer cells in culture. American Journal of Obstetrics and Gynecology 179 6-12. (doi:10.1016/S00029378(98)70244-3)

Petridou E, Mantzoros C, Dessypris N, Koukoulomatis P, Addy C, Voulgaris Z, Chrousos G \& Trichopoulos D 2003 Plasma adiponectin concentrations in relation to endometrial cancer: a case-control study in Greece. Journal of Clinical Endocrinology and Metabolism 88 993-997. (doi:10.1210/jc.2002-021209)

Phalitakul S, Okada M, Hara Y \& Yamawaki H 2011 Vaspin prevents TNF$\alpha$-induced intracellular adhesion molecule- 1 via inhibiting reactive oxygen species-dependent NF- $\kappa \mathrm{B}$ and PKC $\theta$ activation in cultured rat vascular smooth muscle cells. Pharmacological Research 64 493-500. (doi:10.1016/j.phrs.2011.06.001)

Rutanen EM, Nyman T, Lehtovirta P, Ammala M \& Pekonen F 1994 Suppressed expression of insulin-like growth factor binding protein-1 mRNA in the endometrium: a molecular mechanism associating endometrial cancer with its risk factors. International Journal of Cancer 59 307-312. (doi:10.1002/ijc.2910590303)

Schmandt RE, Iglesias DA, Co NN \& Lu KH 2011 Understanding obesity and endometrial cancer risk: opportunities for prevention. American Journal of Obstetrics and Gynecology 205 518-525. (doi:10.1016/j.ajog. 2011.05.042)

Siegel R, Naishadham D \& Jemal A 2013 Cancer statistics, 2013. CA: A Cancer Journal for Clinicians 63 11-30. (doi:10.3322/caac.21166)

Soliman PT, Wu D, Tortolero-Luna G, Schmeler KM, Slomovitz BM, Bray MS, Gershenson DM \& Lu KH 2006 Association between adiponectin, insulin resistance, and endometrial cancer. Cancer 106 2376-2381. (doi:10.1002/cncr.21866)

Takahashi M, Arita Y, Yamagata K, Matsukawa Y, Okutomi K, Horie M, Shimomura I, Hotta K, Kuriyama H, Kihara S et al. 2000 Genomic structure and mutations in adipose-specific gene, adiponectin. International Journal of Obesity and Related Metabolic Disorders 24 861-868. (doi:10.1038/sj.ijo.0801244)

Wallace TM, Levy JC \& Matthews DR 2004 Use and abuse of HOMA modeling. Diabetes Care 27 1487-1495. (doi:10.2337/diacare.27. 6.1487)

Wang Y, Xu A, Knight C, Xu LY \& Cooper GJ 2002 Hydroxylation and glycosylation of the four conserved lysine residues in the collagenous domain of adiponectin. Potential role in the modulation of its insulinsensitizing activity. Journal of Biological Chemistry 277 19521-19529. (doi:10.1074/jbc.M200601200)

Yamauchi T, Kamon J, Waki H, Terauchi Y, Kubota N, Hara K, Mori Y, Ide T, Murakami K, Tsuboyama-Kasaoka N et al. 2001 The fat-derived hormone adiponectin reverses insulin resistance associated with both lipoatrophy and obesity. Nature Medicine 7 941-946. (doi:10.1038/ 90984)

Yamauchi N, Takazawa Y, Maeda D, Hibiya T, Tanaka M, Iwabu M, Okada-Iwabu M, Yamauchi T, Kadowaki T \& Fukayama M 2012 Expression levels of adiponectin receptors are decreased in human endometrial adenocarcinoma tissues. International Journal of Gynecological Pathology 31 352-357. (doi:10.1097/PGP.0b013e3182469583)

Received in final form 26 July 2013

Accepted 19 July 2013

Made available online as an Accepted Preprint

29 July 2013 http://erc.endocrinology-journals.org

DOI: $10.1530 /$ ERC-13-0280 (c) 2013 Society for Endocrinology Printed in Great Britain 